\section{Omalizumab reduziert IgE-Produktion}

\author{
Omalizumab ist ein monoklonaler Anti-lgE-Antikörper, der an zirku- \\ lierende IgE-Moleküle bindet und die Exprimierung des hochaffinen \\ IgE-Rezeptors auf Basophilen und Mastzellen unterdrückt. Sind noch \\ weitere Mechanismen an seiner Wirkung beteiligt?
}

\begin{abstract}
K linisch wird Omalizumab aktuell als Add-on-Therapie bei schwerem und schlecht kontrollierbarem allergischen Asthma bronchiale eingesetzt. $\mathrm{Ob}$ der Antikörper bei diesen Patienten auch die IgE-Produktion beeinflusst, war bisher nicht bekannt. Berliner Allergologen rekrutierten zur Beantwortung dieser Frage 19 Patienten mit mäßigem bis schwerem Asthma bronchiale. Diese erhielten zusätzlich zu ihrer Standardmedikation randomisiert und doppelblind subkutan entweder Omalizumab oder
\end{abstract}

Plazebo in zwei- oder vierwöchigen Intervallen. Die Dosierung von Omalizumab erfolgte abhängig von Körpergewicht und IgE-Spiegel. Gemessen wurde die IgE-Produktion von peripheren mononukleären Blutzellen nach In-vitroStimulation mit IL-4 und CD40-Ligand. Weiter wurde der zelluläre Immunstatus durchflusszytometrisch bestimmt.

Die Antikörperbehandung reduzierte die stimulierte IgE-Freisetzung im Vergleich zu Plazebo deutlich $(\mathrm{p}<0,05)$. Zwölf Wochen nach Beendigung der Be- handlung war die Zahl der zirkulierenden B-Lymphozyten bei den mit Verum behandelten Patienten niedriger als bei den Patienten unter Plazebo $(\mathrm{p}<0,01)$. Bei den anderen Lymphozyten-Subpopulationen zeigten sich keine Unterschiede. Wie Omalizumab diese regulativen Effekte herbeiführt, ist noch nicht klar.

Fazit: Die Behandlung mit dem AntiIgE-Antikörper Omalizumab führt zu einem Rückgang der IgE-Sekretion und der zirkulierenden B-Lymphozyten. Diese Effekte weisen auf eine antiinflammatorische Potenz des Antikörpers hin und könnten auch die Basis für Dosisreduktionschemata liefern.

Hanf $\mathrm{G}$ et al. Omalizumab decreased IgErelease and induced changes in cellular immunity in patients with allergic asthma. Allergy 2007; 61: 1141-4

\title{
Anti-IgE-Therapie und Helminthose-Risiko
}

\section{Bei parasitären Erkrankungen, insbesondere bei Wurminfektionen, zeigen sich meist erhöhte IgE-Spiegel, deren Bedeutung kontrovers diskutiert wird. Brasilianische Forscher gingen jetzt der Frage nach, ob eine IgE-Blockade mit dem Antikörper Omalizumab möglicher- weise die Häufigkeit von Helminthosen beeinflusst.}

B rasilianische Forscher untersuchten im Rahmen einer randomisierten Studie 137 junge Patienten, die an allergischem Asthma oder perennialer Rhinitis litten. Die Studienteilnehmer hatten zudem entweder aktuell eine Helminthose (Ascaris lumbricoides, Trichuris trichiura, Ancylostoma duodenale, Strongyloides stercoralis, Enterobius vermicularis) oder wurden als Risikopatienten eingestuft. Letztere hatten im Jahr zuvor eine Wurmerkrankung durchgemacht, lebten in Gemeinschaft mit einem Parasitenträger oder wiesen hohe spezifische Anti-Askaris-IgE-Antikörper auf. Bei allen Patienten wurde eine Breitspektrumtherapie gegen Würmer durchgeführt. Anschließend erhielten sie entsprechend den Dosierungsempfehlungen Omalizumab oder Plazebo über ein Jahr.

Von den Patienten, die Omalizumab erhalten hatten, entwickelten
50\% im Beobachtungszeitraum mindestens eine intestinale Helminthose. In der Plazebogruppe zeigte sich bei $41 \%$ eine Infektion. Dieser Unterschied war statistisch nicht signifikant. Allerdings waren in der Verumgruppe um 12\% weniger Studienteilnehmer mit zuvor

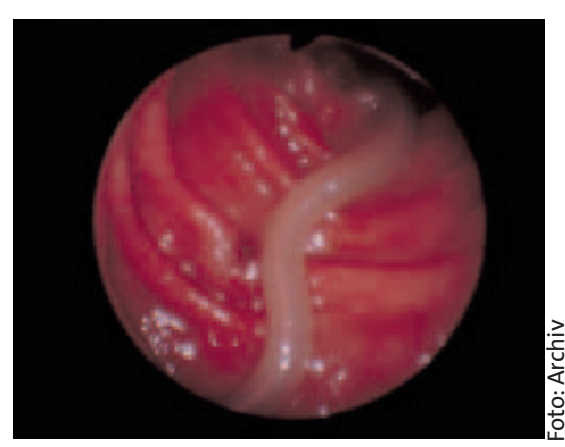

Treten Wurminfektionen wie hier durch Ascaris lumbricoides unter Anti-lgETherapie häufiger auf? aktiver Helminthose als in der Plazebogruppe. Unter Berücksichtigung dieses Infektionsstatus sowie von Geschlecht und Alter der Patienten ergab sich eine Odds Ratio von 2,2 (95\%-Konfidenzintervall $0,94-5,15 ; p=0,03)$ für eine Wurminfektion unter Omalizumab im Verleich zur Plazebogruppe. Die Zeitintervalle von Studienbeginn bis zur ersten Infektion unterschieden sich in den beiden Patientengruppen nicht signifikant.

Die immerhin einjährige Behandlung mit dem Anti-IgE-Antikörper führte nicht zu schwereren Erkrankungen, was anhand von klinischem Aspekt, Laborbefunden, Krankeitsintensität und Notwendigkeit zusätzlicher Interventionen beurteilt wurde.

Fazit: Bei Patienten mit hohem Helminthoserisiko scheint die Therapie mit Omalizumab die Infektionswahrscheinlichkeit geringfügig zu erhöhen. Die Erkrankungen verlaufen jedoch nicht schwerer als unter Plazebo.

Cruz AA et al. Safety of anti-immunoglobulin E therapy with omalizumab in allergic patients at risk of geohelminth infection. Clin Exp Allergy 2007; 37: 197207 